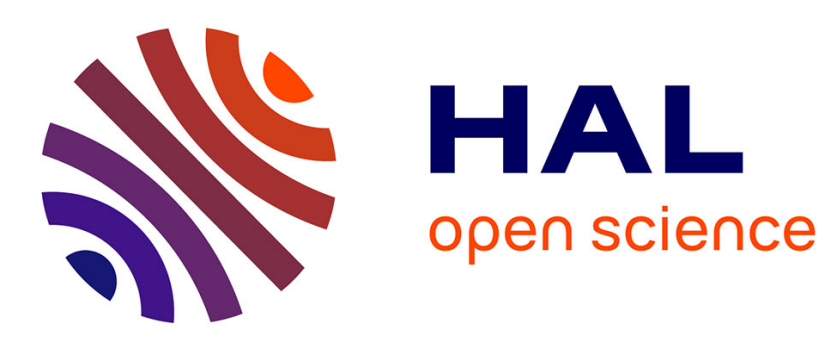

\title{
Riemannian Mathematical Morphology
}

Jesus Angulo, Santiago Velasco-Forero

\section{To cite this version:}

Jesus Angulo, Santiago Velasco-Forero. Riemannian Mathematical Morphology. 2013. hal$00877144 \mathrm{v} 1$

HAL Id: hal-00877144

https://hal-mines-paristech.archives-ouvertes.fr/hal-00877144v1

Preprint submitted on 28 Oct 2013 (v1), last revised 17 Jan 2016 (v3)

HAL is a multi-disciplinary open access archive for the deposit and dissemination of scientific research documents, whether they are published or not. The documents may come from teaching and research institutions in France or abroad, or from public or private research centers.
L'archive ouverte pluridisciplinaire HAL, est destinée au dépôt et à la diffusion de documents scientifiques de niveau recherche, publiés ou non, émanant des établissements d'enseignement et de recherche français ou étrangers, des laboratoires publics ou privés. 


\title{
Riemannian Mathematical Morphology
}

\author{
Jesús Angulo $^{\mathrm{a}}$, Santiago Velasco-Forero ${ }^{\mathrm{b}}$ \\ ${ }^{a}$ MINES ParisTech, CMM - Centre de Morphologie Mathématique, 35 rue St Honoré 77305 Fontainebleau Cedex, France \\ ${ }^{b}$ National University of Singapore, Department of Mathematics.
}

\begin{abstract}
This paper introduces mathematical morphology operators for real-valued images whose support space is a Riemannian manifold. The starting point consists in replacing the Euclidean distance in the canonic quadratic structuring function by the Riemannian distance used for the adjoint dilation/erosion. We then extend the canonic case to a most general framework of Riemannian operators based on the notion of admissible Riemannian structuring function. An alternative paradigm of morphological Riemannian operators involves an external structuring function which is parallel transported to each point on the manifold. Besides the definition of the various Riemannian dilation/erosion and Riemannian opening/closing, their main properties are studied. We generalize also some results on Lasry-Lions regularization for non-smooth images on Cartan-Hadamard manifolds. Theoretical connections with previous works on adaptive morphology and manifold shape morphology are also considered. From a practical viewpoint, various useful image embedding into Riemannian manifolds are formalized, with some illustrative examples of morphological processing real-valued 3D surfaces.
\end{abstract}

Keywords: mathematical morphology, manifold nonlinear image processing, Riemannian images, Riemannian image embedding, Riemannian structuring function, morphological processing of surfaces

\section{Introduction}

Pioneered for Boolean random sets (Matheron, 1975), extended latter to grey-level images (Serra, 1982) and more generally formulated in the framework of complete lattices (Serra, 1988; Heijmans, 1994), mathematical morphology is a nonlinear image processing methodology useful for solving efficiently many image analysis tasks (Soille, 1999). Our motivation in this paper is to formulate morphological operators for scalar functions on curved spaces.

Let $E$ be the Euclidean $\mathbb{R}^{d}$ or discrete space $\mathbb{Z}^{d}$ (support space) and let $\mathcal{T}$ be a set of grey-levels (space of values). It is assumed that $\mathcal{T}=\overline{\mathbb{R}}=\mathbb{R} \cup\{-\infty,+\infty\}$. A grey-level image is represented by a function $f: E \rightarrow \mathcal{T}, f \in \mathcal{F}(E, \mathcal{T})$, i.e., $f$ maps each pixel $x \in E$ into a grey-level value in $\mathcal{T}$. Given a greylevel image, the two basic morphological mappings $\mathcal{F}(E, \mathcal{T}) \rightarrow$ $\mathcal{F}(E, \mathcal{T})$ are the dilation and the erosion given respectively by

$$
\left\{\begin{array}{l}
\delta_{b}(f)(x)=(f \oplus b)(x)=\sup _{y \in E}\{f(y)+b(y-x)\}, \\
\varepsilon_{b}(f)(x)=(f \ominus b)(x)=\inf _{y \in E}\{f(y)-b(y+x)\},
\end{array}\right.
$$

where $b \in \mathcal{F}(E, \mathcal{T})$ is the structuring function which determines the effect of the operator. By allowing infinity values, the further convention for ambiguous expressions should be considered: $f(y)+b(x-y)=-\infty$ when $f(y)=-\infty$ or $b(x-y)=-\infty$, and that $f(y)-b(y+x)=+\infty$ when $f(y)=+\infty$ or $b(y+x)=-\infty$. We easily note that both are invariant under translations in the spatial ("horizontal") space $E$ and in the grey-level ("vertical")

Email addresses: jesus. angulo@mines-paristech.fr (Jesús Angulo), matsavf@nus.edu.sg (Santiago Velasco-Forero) space $\mathcal{T}$, i.e., $f(x) \mapsto f_{h, \alpha}(x)=f(x-h)+\alpha, x \in E$ and $\alpha \in \mathbb{R}$, then $\delta_{b}\left(f_{h, \alpha}\right)(x)=\delta_{b}(f)(x-h)+\alpha$. The other morphological operators, such as the opening and the closing, are obtained by composition of dilation/erosion (Serra, 1982; Heijmans, 1994).

The structuring function is usually a parametric multi-scale family (Jackway and Deriche, 1996) $b_{\lambda}(x)$, where $\lambda>0$ is the scale parameter such that $b_{\lambda}(x)=\lambda b(x / \lambda)$ and which satisfies the semi-group property $\left(b_{\lambda} \oplus b_{\mu}\right)(x)=b_{\lambda+\mu}(x)$. It is well known in the state-of-the-art of Euclidean morphology that the canonic family of structuring functions is the quadratic (or parabolic) one (Maragos, 1995; van den Boomgaard and Dorst, 1997); i.e.,

$$
b_{\lambda}(x)=q_{\lambda}(x)=-\frac{\|x\|^{2}}{2 \lambda} .
$$

The most commonly studied framework, which additionally presents better properties of invariance, is based on flat structuring functions, called structuring elements. More precisely, let $B$ be a Boolean set defined at the origin, i.e., $B \subseteq E$ or $B \in \mathcal{P}(E)$, which defines the "shape" of the structuring element, the associated structuring function is given by

$$
b(x)=\left\{\begin{array}{cc}
0 & \text { if } x \in B \\
-\infty & \text { if } x \in B^{c}
\end{array}\right.
$$

where $B^{c}$ is the complement set of $B$ in $\mathcal{P}(E)$. Hence, the flat dilation and flat erosion can be computed respectively by the moving local maxima and minima filters.

Aim of the paper. Let us consider now that the support space is not Euclidean, see Fig. 1(a). This is the case for instance if we deal with a smooth 3D surface, or more generally if the support 
space is a Riemannian manifold. The present work was inspired by the idea of Riemannian inf-convolution introduced in (Azagra and Ferrera, 2006), which replaced the Euclidean distance in the canonic quadratic structuring function by the Riemannian distance. We adopt exactly the same starting point for the formulation of dilation/erosion in Riemannian manifolds (Section 3). Besides the definition of Riemannian dilation/erosion and Riemannian opening/closing, we explore their properties and in particular the associated granulometric scale-space. We also generalize some theoretical results on Lasry-Lions regularization, with an original result for Cartan-Hadamard manifolds. We then extend the canonic case to the most general framework of Riemannian dilation/erosion and subsequent operators in Section 4, by introducing the notion of admissible Riemannian structuring function. Section 5 introduces a different paradigm of morphological operators on Riemannian supported images, where the structuring function is an external datum which is parallel transported to each point on the manifold. We consider theoretically various useful case studies of image manifolds in Section 7, but due to the limited paper length, we only illustrate some cases of real-valued 3D surfaces.

This paper is an extended and completed version of the ISMM'13 conference paper (Angulo and Velasco-Forero, 2013); however, again by limited length, we have skipped the proofs of all the theoretical results, some of them available on (Angulo and Velasco-Forero, 2013).

Related work. Generalizations of Euclidean translation-invariant morphology have followed three main directions. On the one hand, adaptive morphology (Debayle and Pinoli, 2005; Lerallut et al., 2007; Welk et al., 2011; Verdú et al., 2011; Ćurić et al., 2012; Angulo, 2013; Landström and Thurley, 2013; VelascoForero and Angulo, 2013), where the structuring function becomes dependent on the position or the input image itself. Section 6 explores the connections of our framework with such kind of approaches. On the second hand, group morphology (Roerdink, 2000), where the translation invariance is replaced by other group invariance (similarity, affine, spherical, projective, etc.). Related to that, we have also the morphology for binary manifolds (Roerdink, 1994), whose relationship with our formulation is deeply studied in Section 5. Finally, we should cite also the classical notion of geodesic dilation (Lantuejoul and Beucher, 1981) as the basic operator for (connective) geodesic reconstruction (Soille, 1999), where the marker image is dilated according to the metric yielded by the reference image (see also Section 6).

\section{Basics on Riemannian manifold geometry}

Let us remind in this section some basics on differential geometry for Riemannian manifolds (Berger and Gostiaux, 1987), see Fig. 1(b) for a explanatory diagram.

The tangent space of the manifold $\mathcal{M}$ at a point $p \in \mathcal{M}$, denoted by $T_{p} \mathcal{M}$, is the set of all vectors tangent to $\mathcal{M}$ at $p$. The first issue to consider is how to transport vectors from one point of $\mathcal{M}$ to another. Let $p, q \in \mathcal{M}$ and let $\gamma:[a, b] \rightarrow \mathcal{M}$ be a parameterized curve (or path) from $\gamma(a)=p$ to $\gamma(b)=q$. For (a)

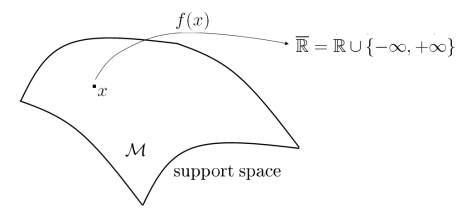

(b)

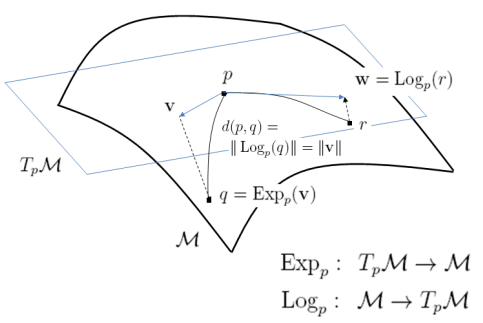

Figure 1: (a) Real-valued Riemannian image. (b) Riemannian manifold at tangent space a given point.

$\mathbf{v} \in T_{p} \mathcal{M}$, let $\mathbf{V}$ be the unique parallel vector field along $\gamma$ with $\mathbf{V}(a)=\mathbf{v}$. The map $P_{\gamma}: T_{p} \mathcal{M} \rightarrow T_{q} \mathcal{M}$ determined by $P_{\gamma}(\mathbf{v})=$ $\mathbf{V}(b)$ is called parallel transport from $p$ to $q$ along $\gamma$, and $P_{\gamma}(\mathbf{v})$ the parallel translate of $v$ along $\gamma$ to $q$. Note that parallel transport from $p$ to $q$ is path dependent: the difference between two paths is a rotation around the normal to $\mathcal{M}$ at $q$. The Riemannian distance between two points $p, q \in \mathcal{M}$, denoted $d(p, q)$, is defined as the minimum length over all possible smooth curves between $p$ and $q$. A geodesic $\gamma:[0,1] \rightarrow \mathcal{M}$ connecting two points $p, q \in \mathcal{M}$ is the shortest path on $\mathcal{M}$ having elements $p$ and $q$ as endpoints. The geodesic curve $\gamma(t)$ can be specified in terms of a starting point $p \in \mathcal{M}$ and a tangent vector (initial constant velocity) $\mathbf{v} \in T_{p} \mathcal{M}$ as it represents the solution of Christoffel differential equation with boundary conditions $\gamma(0)=p$ and $\dot{\gamma}(0)=\mathbf{v}$. The idea behind exponential map $\operatorname{Exp}_{p}$ is to parameterize a Riemannian manifold $\mathcal{M}$, locally near any $p \in \mathcal{M}$, in terms of a mapping from the tangent space $T_{p} \mathcal{M}$ into a point in $\mathcal{M}$. The exponential map is injective on a zero-centered ball $B$ in $T_{p} \mathcal{M}$ of some non-zero (possibly infinity) radius. Thus for a point $q$ in the image of $B$ under $\operatorname{Exp}_{p}$ there exists a unique vector $\mathbf{v} \in T_{p} \mathcal{M}$ corresponding to a minimal length path under the exponential map from $p$ to $q$. Exponential maps may be associated to a manifold by the help of geodesic curves. The exponential map $\operatorname{Exp}_{p}: T_{p} \mathcal{M} \rightarrow \mathcal{M}$ associated to any geodesic $\gamma_{\mathbf{v}}$ emanating from $p$ with tangent at the origin $\mathbf{v} \in T_{p} \mathcal{M}$ is defined as $\operatorname{Exp}_{p}(\mathbf{v})=\gamma_{\mathbf{v}}(1)$, where the geodesic is given by $\gamma_{\mathbf{v}}(t)=\operatorname{Exp}_{p}(t \mathbf{v})$. The geodesic has constant speed equal to $\left\|d \gamma_{\mathbf{v}} / d t\right\|(t)=\|\mathbf{v}\|$, and thus the exponential map preserves distances for the initial point: $d\left(p, \operatorname{Exp}_{p}(\mathbf{v})\right)=\|\mathbf{v}\|$. A Riemannian manifold is geodesically complete if and only if the exponential map $\operatorname{Exp}_{p}(\mathbf{v})$ is defined $\forall p \in \mathcal{M}$ and $\forall \mathbf{v} \in T_{p} \mathcal{M}$. The inverse operator, named logarithm map, $\operatorname{Exp}_{p}^{-1}=\log _{p}$ maps a point of $q \in \mathcal{M}$ into to their associated tangent vectors $\mathbf{v} \in T_{p} \mathcal{M}$. The exponential map is in general only invertible for a sufficient small neighbourhood of the origin in $T_{p} \mathcal{M}$, although on some manifolds the inverse exists for arbitrary neighbourhoods. For a point $q$ in the domain of $\log _{p}$ the geodesic distance between $p$ and $q$ is given by $d(p, q)=\left\|\log _{p}(q)\right\|$. 


\section{Canonic Riemannian dilation and erosion}

Let us start by a formal definition of the two basic canonic morphological operators for images supported on a Riemannian manifold.

Definition 1. Let $\mathcal{M}$ a complete Riemannian manifold and $d_{\mathcal{M}}$ : $\mathcal{M} \times \mathcal{M} \rightarrow \mathbb{R}^{+},(x, y) \mapsto d_{\mathcal{M}}(x, y)$, is the geodesic distance on $\mathcal{M}$, for any image $f: \mathcal{M} \rightarrow \overline{\mathbb{R}}, \overline{\mathbb{R}}=\mathbb{R} \cup\{-\infty,+\infty\}$, so $f \in \mathcal{F}(\mathcal{M}, \overline{\mathbb{R}})$ and for $\lambda>0$ we define for every $x \in \mathcal{M}$ the canonic Riemannian dilation of $f$ of scale parameter $\lambda$ as

$$
\delta_{\lambda}(f)(x)=\sup _{y \in \mathcal{M}}\left\{f(y)-\frac{1}{2 \lambda} d_{\mathcal{M}}(x, y)^{2}\right\}
$$

and the canonic Riemannian erosion of $f$ of parameter $\lambda$ as

$$
\varepsilon_{\lambda}(f)(x)=\inf _{y \in \mathcal{M}}\left\{f(y)+\frac{1}{2 \lambda} d_{\mathcal{M}}(x, y)^{2}\right\}
$$

An obvious property of the canonic Riemannian dilation and erosion is the duality by the involution $f(x) \mapsto \complement f(x)=$ $-f(x)$, i.e., $\delta_{\lambda}(f)=\complement \varepsilon_{\lambda}(\complement f)$. As in classical Euclidean morphology, the adjunction relationship is fundamental for the construction of the rest of morphological operators.

Proposition 2. For any two real-valued images defined on the same Riemannian manifold $\mathcal{M}$, i.e., $f, g: \mathcal{M} \rightarrow \overline{\mathbb{R}}$, the pair $\left(\varepsilon_{\lambda}, \delta_{\lambda}\right)$ is called the canonic Riemannian adjunction

$$
\delta_{\lambda}(f)(x) \leq g(x) \Leftrightarrow f(x) \leq \varepsilon_{\lambda}(g)(x)
$$

Hence, we have an adjunction if both images $f$ and $g$ are defined on the same Riemannian manifold $\mathcal{M}$, or in other terms, when the same "quadratic geodesic structuring function":

$$
\mathfrak{q}_{\lambda}(x ; y)=-\frac{1}{2 \lambda} d_{\mathcal{M}}(x, y)^{2},
$$

is considered for pixel $x \mapsto \mathfrak{q}_{\lambda}(x ; y), y \in \mathcal{M}$ in both $f$ and $g$. This result implies in particular that the canonic Riemannian dilation commutes with the supremum and the dual erosion with the infimum, i.e., for a given collection of images $f_{i} \in \mathcal{F}(\mathcal{M}, \overline{\mathbb{R}}), i \in I$, we have

$$
\delta_{\lambda}\left(\bigvee_{i \in I} f_{i}\right)=\bigvee_{i \in I} \delta_{\lambda}\left(f_{i}\right) ; \quad \varepsilon_{\lambda}\left(\bigwedge_{i \in I} f_{i}\right)=\bigwedge_{i \in I} \varepsilon_{\lambda}\left(f_{i}\right) .
$$

In addition, using the classical result on adjunctions in complete lattices (Heijmans, 1994), we state that the composition products of the pair $\left(\varepsilon_{\lambda}, \delta_{\lambda}\right)$ lead to the adjoint opening and adjoint closing if and only the field of geodesic structuring functions is computed on a common manifold $\mathcal{M}$.

Definition 3. Given an image $f \in \mathcal{F}(\mathcal{M}, \overline{\mathbb{R}})$, the canonic Riemannian opening and canonic Riemannian closing of scale parameter $\lambda$ are respectively given by

$$
\gamma_{\lambda}(f)(x)=\sup _{z \in \mathcal{M}} \inf _{y \in \mathcal{M}}\left\{f(y)+\frac{1}{2 \lambda} d_{\mathcal{M}}(z, y)^{2}-\frac{1}{2 \lambda} d_{\mathcal{M}}(z, x)^{2}\right\},
$$

and

$$
\varphi_{\lambda}(f)(x)=\inf _{z \in \mathcal{M}} \sup _{y \in \mathcal{M}}\left\{f(y)-\frac{1}{2 \lambda} d_{\mathcal{M}}(z, y)^{2}+\frac{1}{2 \lambda} d_{\mathcal{M}}(z, x)^{2}\right\} .
$$

This technical point is very important since in some image manifold embedding the Riemannian manifold support $\mathcal{M}$ of image $f$ depends itself on $f$. If $\mathcal{M}$ does not depends on $f$, the canonic Riemannian opening and closing are respectively given by $\gamma_{\lambda}(f)=\delta_{\lambda}\left(\varepsilon_{\lambda}(f)\right)$, and $\varphi_{\lambda}(f)=\varepsilon_{\lambda}\left(\delta_{\lambda}(f)\right)$. We notice that this issue was already considered by Roerdink (2009) for the case of adaptive neighbourhood morphology.

Having the canonic Riemannian opening and closing, all the other morphological filters defined by composition of them are easily obtained.

\subsection{Properties of $\delta_{\lambda}(f)$ and $\varepsilon_{\lambda}(f)$}

Classical properties of Euclidean dilation and erosion have also the equivalent for Riemannian manifold $\mathcal{M}$, and they do not dependent on the geometry of $\mathcal{M}$.

Proposition 4. Let $\mathcal{M}$ be a Riemannian manifold, and let $f, g \in$ $\mathcal{F}(\mathcal{M}, \overline{\mathbb{R}})$ two real valued images $\mathcal{M}$. We have the following properties for the canonic Riemannian operators.

1. (Increaseness) If $f(x) \leq g(x), \forall x \in \mathcal{M}$ then $\delta_{\lambda}(f)(x) \leq$ $\delta_{\lambda}(g)(x)$ and $\varepsilon_{\lambda}(f)(x) \leq \varepsilon_{\lambda}(g)(x), \forall x \in \mathcal{M}$ and $\forall \lambda>0$.

2. (Extensivity and anti-extensivity) $\delta_{\lambda}(f)(x) \geq f(x)$ and $\varepsilon_{\lambda}(f)(x) \leq f(x), \forall x \in \mathcal{M}$ and $\forall \lambda>0$.

3. (Ordering property) If $0<\lambda_{1}<\lambda_{2}$ then $\delta_{\lambda_{2}}(f)(x) \geq$ $\delta_{\lambda_{1}}(f)(x)$ and $\varepsilon_{\lambda_{2}}(f)(x) \leq \varepsilon_{\lambda_{1}}(f)(x)$.

4. (Invariance under isometry) If $T: \mathcal{M} \rightarrow \mathcal{M}$ is an isometry of $\mathcal{M}$ and if $f$ is invariant under $T$, i.e., $f(T z)=f(z)$ for all $z \in \mathcal{M}$, then the Riemannian dilation and erosion are also invariant under $T$, i.e., $\delta_{\lambda}(f)(T z)=\delta_{\lambda}(f)(z)$ and $\varepsilon_{\lambda}(f)(T z)=\varepsilon_{\lambda}(f)(z), \forall z \in \mathcal{M}$ and $\forall \lambda>0$.

5. (Extrema preservation) We have $\sup \delta_{\lambda}(f)=\sup f$ and $\inf \varepsilon_{\lambda}(f)=\inf f$, moreover if $f$ is lower (resp. upper) semicontinuous then every minimizer (resp. maximizer) of $\varepsilon_{\lambda}(f)\left(\right.$ resp. $\left.\delta_{\lambda}(f)\right)$ is a minimizer (resp. maximizer) of $f$, and conversely.

\subsection{Flat isotropic Riemannian dilation and erosion}

In order to obtain the counterpart of flat isotropic Euclidean dilation and erosion, we replace the quadratic structuring function $\mathfrak{q}_{\lambda}(x, y)$ by a flat structuring function given by the geodesic ball of radius $r$ centered at $x$, i.e.,

$$
B_{r}(x)=\left\{y: d_{\mathcal{M}}(x, y) \leq r\right\}, r>0 .
$$

The corresponding flat isotropic Riemannian dilation and erosion of size $r$ are given by:

$$
\begin{aligned}
& \delta_{B_{r}}(f)(x)=\sup \left\{f(y): y \in \check{B}_{r}(x)\right\}, \\
& \varepsilon_{B_{r}}(f)(x)=\inf \left\{f(y): y \in B_{r}(x)\right\} .
\end{aligned}
$$

where $\breve{B}_{r}(x)$ is the transposed shape of ball $B_{r}(x)$. Corresponding flat isotropic Riemannian opening and closing are obtained by composition of operators (8) and (9):

$$
\gamma_{B_{r}}(f)=\delta_{B_{r}}\left(\varepsilon_{B_{r}}(f)\right) ; \varphi_{B_{r}}(f)=\varepsilon_{B_{r}}\left(\delta_{B_{r}}(f)\right) .
$$

All the properties formulated for canonic operators hold for flat isotropic ones too. For practical applications, it should be noted that flat operators typically lead to stronger filtering effects than the quadratic ones. 


\subsection{Riemannian granulometries:}

scale-space properties of $\gamma_{\lambda}(f)$ and $\varphi_{\lambda}(f)$

For the canonic Riemannian opening and closing, we have also the classical properties which are naturally proved as a consequence of the adjunction, see (Heijmans, 1994).

Proposition 5. Let $\gamma_{\lambda}(f)$ and $\varphi_{\lambda}(f)$ be respectively the canonic Riemannian opening and closing of an image $f \in \mathcal{F}(\mathcal{M}, \overline{\mathbb{R}})$.

1. $\gamma_{\lambda}(f)$ and $\varphi_{\lambda}(f)$ are both increasing operators.

2. $\gamma_{\lambda}(f)$ is anti-extensive and $\varphi_{\lambda}(f)$ extensive with the following ordering relationships, i.e., for for $0<\lambda_{1} \leq \lambda_{2}$, we have:

$$
\gamma_{\lambda_{2}}(f)(x) \leq \gamma_{\lambda_{1}}(f)(x) \leq f(x) \leq \varphi_{\lambda_{1}}(f)(x) \leq \varphi_{\lambda_{2}}(f)(x) ;
$$

3. idempotency of both operators, $\gamma_{\lambda}\left(\gamma_{\lambda}(f)\right)=\gamma_{\lambda}(f)$ and $\varphi_{\lambda}\left(\varphi_{\lambda}(f)\right)=\varphi_{\lambda}(f)$

Property 3 on idempotency together with the increaseness defines a family of so-called algebraic openings/closings (Serra, 1988; Heijmans, 1994) larger than the one associated to the composition of dilation/erosion. Idempotent and increasing operators are also known as ethmomorphisms by Kiselman (2007). Anti-extensivity and extensivity involves that $\gamma_{\lambda}$ is a anoiktomorphism and $\varphi_{\lambda}$ a cleistomorphism. One of the most classical results in morphological operators provided us an example of algebraic opening: given a collection of openings $\left\{\gamma_{i}\right\}$, increasing, idempotent and anti-extensive operators for all $i$, the supremum of them $\sup _{i} \gamma_{i}$ is also an opening (Matheron, 1975). A dual result is obtained for the closing by changing the sup by the inf.

The class of openings (resp. closings) is neither closed under infimum (resp. opening) or a generic composition. There is however a semi-group property leading to a scale-space framework for opening/closing operators, known as granulometries. The notion of granulometry in Euclidean morphology is summarized in the following results (Matheron, 1975; Serra, 1988).

Theorem 6 (Matheron (1975), Serra (1988)). A parameterized family $\left\{\gamma_{\lambda}\right\}_{\lambda>0}$ of flat openings from $\mathcal{F}(E, \mathcal{T})$ into $\mathcal{F}(E, \mathcal{T})$ is a granulometry (or size ditritribution) when

$$
\gamma_{\lambda_{1}} \gamma_{\lambda_{2}}=\gamma_{\lambda_{2}} \gamma_{\lambda_{1}}=\gamma_{\sup \left(\lambda_{1}, \lambda_{2}\right)} ; \lambda_{1}, \lambda_{2}>0 .
$$

Condition (12) is equivalent to both

$$
\begin{array}{ll}
\gamma_{\lambda_{1}} \leq \gamma_{\lambda_{2}} ; & \lambda_{1} \geq \lambda_{2}>0 ; \\
\mathcal{B}_{\lambda_{1}} \subseteq \mathcal{B}_{\lambda_{2}} ; & \lambda_{1} \geq \lambda_{2}>0
\end{array}
$$

where $\mathcal{B}_{\lambda}$ is the invariance domain of the opening at scale $\lambda$; i.e., the family of structuring elements Bs such that $B=\gamma_{\lambda}(B)$ (Serra, 1988).

By duality, we introduce antisize distributions as the families of closings $\left\{\varphi_{\lambda}\right\}_{\lambda>0}$.

Axiom (12) shows how translation invariant flat openings are composed and highlights their semi-group structure. Equivalent condition (13) emphasizes the monotonicity of the granulometry with respect to $\lambda$ : the opening becomes more and more active as $\lambda$ increases. When dealing with Euclidean spaces, Matheron (1975) introduced the notion of Euclidean granulometry as the size distribution being translationally invariant and compatible with homothetics, i.e., $\gamma_{\lambda}(f(x))=\lambda \gamma_{1}\left(f\left(\lambda^{-1} x\right)\right)$, where $f \in \mathcal{F}(E, \mathcal{T})$ is an Euclidean grey-level images. More precisely, a family of mappings $\gamma_{\lambda}$ is an Euclidean granulometry if and only if there exist a class $\mathcal{B}^{\prime}$ such that

$$
\gamma_{\lambda}(f)=\bigvee_{B \in \mathcal{B}^{\prime}} \bigvee_{\mu \geq \lambda} \gamma_{\mu B}(f)
$$

Then the domain of invariance $\mathcal{B}_{\lambda}$ are equal to $\lambda \mathcal{B}$, where $\mathcal{B}$ is the class closed under union, translation and homothetics $\geq 1$, which is generated by $\mathcal{B}^{\prime}$. If we reduce the class $\mathcal{B}^{\prime}$ to a single element $B$, the associated size distribution becomes

$$
\gamma_{\lambda}(f)=\bigvee_{\mu \geq \lambda} \gamma_{\mu B}(f)
$$

The following key result simplifies the situation. The size distribution by a compact structuring element $B$ is equivalent to $\gamma_{\lambda}(f)=\gamma_{\lambda B}(f)$ if and only if $B$ is convex. The extension of granulometric theory to non-flat structuring functions was deeply studied in (Kraus et al., 1993). In particular, it was proven that one can build grey-level Euclidean granulometries with one structuring function if and only if this function has a convex compact domain and is constant there (flat function).

We can naturally extend Matheron axiomatic to the general case of openings in Riemannian supported images. We start by giving a result which is valid for families of openings $\left\{\psi_{\lambda}\right\}$ (idempotent and anti-extensive operators) more general than the canonic Riemannian openings.

Proposition 7. Given the set of Riemannian openings $\left\{\psi_{\lambda}\right\}_{\lambda>0}$ indexed according to the positive parameter $\lambda$, but not necessary ordered between them, the corresponding Riemannian granulometry on image $f \in \mathcal{F}(\mathcal{M}, \overline{\mathbb{R}})$ is the family of multiscale openings $\left\{\Gamma_{\lambda}\right\}_{\lambda>0}$ generated as

$$
\Gamma_{\lambda}(f)=\bigvee_{\mu \geq \lambda} \psi_{\mu}(f)
$$

such that the granulometric semi-group law holds for any pair of scales:

$$
\Gamma_{\lambda_{1}}\left(\Gamma_{\lambda_{2}}(f)\right)=\Gamma_{\lambda_{2}}\left(\Gamma_{\lambda_{1}}(f)\right)=\Gamma_{\sup \left(\lambda_{1}, \lambda_{2}\right)}(f) .
$$

In the particular case of canonic Riemannian openings, $\left\{\gamma_{\lambda}\right\}_{\lambda>0}$, we always have $\gamma_{\lambda_{1}} \leq \gamma_{\lambda_{2}}$ if $\lambda_{1} \geq \lambda_{2}>0$. Hence, $\Gamma_{\lambda}(f)=\gamma_{\lambda}$ and consequently $\left\{\gamma_{\lambda}\right\}_{\lambda>0}$ is a granulometry. This is also valid for flat isotropic Riemannian openings.

The Riemannian case closest to Matheron's Euclidean granulometries corresponds to the flat isotropic Riemannian openings $\gamma_{B_{r}}$ associated to a concave quadratic geodesic structuring function $\mathfrak{q}_{\lambda}(x, y)$. Or in other terms, the case of a Riemannian manifold $\mathcal{M}$ where the Riemannian distance is always a convex function, since this fact involves that $B_{r}(x)$ as defined in (7) is a convex set for any $r$ at any $x \in \mathcal{M}$. Obviously, the flat convex Riemannian granulometry $\left\{\gamma_{B_{r}}\right\}_{r>0}$ is not translation invariant but we have that $B_{r_{1}}(x) \subseteq B_{r_{2}}(x)$, for $r_{2} \geq r_{1}$ and for any 
$x \in \mathcal{M}$, which involves a natural sieving selection of features in the neighborhood of any point $x$.

A Riemannian distance function which is convex is not only useful for scale-space properties. As discussed just below, one has powerful results of regularization too.

\subsection{Concavity of $\mathfrak{q}_{\lambda}(x ; y)$ and Lipschitz image regularization using $\left(\varepsilon_{\lambda}, \delta_{\lambda}\right)$}

In the field of convex analysis, Moreau-Yosida regularization (Moreau, 1967) consists in computing a regularized version of a scalar function defined on a vector space, by means of an Euclidean erosion using quadratic structuring function.

As discussed in (Azagra and Ferrera, 2006), the results of Moreau-Yosida regularization are extended to functions on a Cartan-Hadamard manifold. We remind that a Cartan-Hadamard manifold is a simply connected Riemannian manifold $\mathcal{M}$ with sectional curvature $K \leq 0$ (Lang, 1999). In fact, an assumption of non-positive curvature of $\mathcal{M}$ is necessary in order that $d_{\mathcal{M}}$ be uniformly locally convex around the diagonal $\mathcal{M} \times \mathcal{M}$.

Let $\mathcal{M}$ be a finite dimensional Cartan-Hadamard manifold, hence every two points can be connected by a minimizing geodesic. Let $A$ be a closed convex subset of $\mathcal{M}$. Then the distance function to $A, x \mapsto d_{\mathcal{M}}(x, A)$, where $d_{\mathcal{M}}(x, A)=\inf \left\{d_{\mathcal{M}}(x, y): y \in A\right\}$ is $C^{1}$ smooth on $\mathcal{M} \backslash A$ and, moreover, the square of the distance function $x \mapsto d_{\mathcal{M}}(x, A)^{2}$ is $C^{1}$ smooth and convex on all of $M$ (Azagra and Ferrera, 2006). Consequently, if $\mathcal{M}$ is a CartanHadamard manifold, the structuring function $x \mapsto \mathfrak{q}(x, y), \forall y \in$ $\mathcal{M}$, is always a concave function; or equivalently, $-\mathfrak{q}(x, y)$ is a convex function.

In this context, we have the following result (Azagra and Ferrera, 2006).

Theorem 8 (Azagra and Ferrera(2006)). Let $\mathcal{M}$ be a CartanHadamard manifold. Let $f: \mathcal{M} \rightarrow \mathbb{R} \cup\{+\infty\}$ be a lowersemicontinuous convex function. Then, the functions $f_{\lambda}: \mathcal{M} \rightarrow$ $\mathbb{R}$, defined by (the canonic Riemannian erosion)

$$
f_{\lambda}(x)=\varepsilon_{\lambda}(f)(x)=\inf _{y \in \mathcal{M}}\left\{f(y)-\mathfrak{q}_{\lambda}(x, y)\right\}
$$

are convex and 1-Lipschitz continuous for all $\lambda>0$. If $f$ is uniformly continuous on bounded set, $f_{\lambda}$ converges to $f$ uniformly on bounded sets.

As a generalization of the use of Moreau-Yosida regularization, the Lasry-Lions regularization (Lasry and Lions, 1986) is a theory of nonsmooth approximation for functions in Hilbert spaces using combinations of Euclidean dilation and erosion with quadratic structuring functions, which leads to the approximation of lower or upper-semicontinuous functions by Lipschitz continuous functions, without assuming convexity of $f$. We propose the following result which generalizes the case of convex functions in Cartan-Hadamard manifolds studied by Azagra and Ferrera (2006) using the results for Hilbert spaces by Attouch and Aze (1993) for the Lasry-Lions regularization.

Theorem 9. Let $\mathcal{M}$ be a Riemannian manifold simply connected and with sectional curvature $K \leq 0$. Given two images $f, g \in$
$\mathcal{F}(\mathcal{M}, \overline{\mathbb{R}})$, for all $0<\mu<\lambda$ let us define the Lasry-Lions regularizers:

$$
\begin{aligned}
& \left(f_{\lambda}\right)^{\mu}(x)=\sup _{z \in \mathcal{M}} \inf _{y \in \mathcal{M}}\left\{f(y)+\frac{1}{2 \lambda} d_{\mathcal{M}}(z, y)^{2}-\frac{1}{2 \mu} d_{\mathcal{M}}(z, x)^{2}\right\} \\
& \left(g^{\lambda}\right)_{\mu}(x)=\inf _{z \in \mathcal{M}} \sup _{y \in \mathcal{M}}\left\{g(y)-\frac{1}{2 \lambda} d_{\mathcal{M}}(z, y)^{2}+\frac{1}{2 \mu} d_{\mathcal{M}}(z, x)^{2}\right\}
\end{aligned}
$$

We have $\left(f_{\lambda}\right)^{\mu} \leq f$ and $\left(g^{\lambda}\right)_{\mu} \geq g$. Assume that there exists $c, d>0$, such that we have the following growing conditions:

$$
f(x) \geq-\frac{c}{2}\left(1+d\left(x, x_{0}\right)^{2}\right), \quad g(x) \leq \frac{d}{2}\left(1+d\left(x, x_{0}\right)^{2}\right), \quad x_{0} \in \mathcal{M}
$$

Then, for all $0<\mu<\lambda<\frac{1}{c}$ the pseudo-opened image $\left(f_{\lambda}\right)^{\mu}$ is a $C^{1}$ function, for all $0<\mu<\lambda<\frac{1}{d}$ the pseudo-closed image $\left(g^{\lambda}\right)_{\mu}$ is a $C^{1}$ function, whose gradient is Lipschitz continuous with constant $\max (1 / \mu, 1 /(\lambda-\mu))$. The image $\left(f_{\lambda}\right)^{\mu}$ is $a \mu^{-1}$-weakly convex function and $a(\lambda-\mu)^{-1}$-weakly concave function.

In addition, if $f$ is a lower (resp. $g$ is a upper) semicontinuous function, the pseudo-opening of $f$ (resp. pseudo-closing of g) converges uniformly to $f$ (resp. $g$ ); i.e., $\lim _{\lambda, \mu \rightarrow 0}\left(f_{\lambda}\right)^{\mu}(x)=$ $f(x)$ and $\lim _{\lambda, \mu \rightarrow 0}\left(g^{\lambda}\right)_{\mu}(x)=g(x)$.

As we can see, the convexity/concavity of the function $f$ in Theorem 8 has been replaced by some geodesically quadratically minorized $f(x) \geq-\frac{c}{2}\left(1+d\left(x, x_{0}\right)^{2}\right)$ and majorized $g(x) \leq$ $\frac{d}{2}\left(1+d\left(x, x_{0}\right)^{2}\right)$ assumptions. The idea is that given a quadratically majorized function $g$ in $\mathcal{M}$ of parameter $d$, the quadratic dilation $\delta_{\lambda}$ with $\lambda<c^{-1}$ produces a kind of $\lambda$-weakly convex function. Then for any $\mu<\lambda$ (strictly smaller than the dilation scale), the corresponding quadratic erosion $\varepsilon_{\mu}$ produces a function belongings to the class of bounded $C^{1}$, with a Lipschitz continuous gradient.

We notice that this theoretically valid only for manifolds of nonpositive sectional curvature; the canonic example of negative curvature manifold is the hyperboloid; conversely, the typical case of positively curved Riemannian manifold is the sphere, i.e., there are closed convex sets $C$ of arbitrarily small diameter in $\mathbb{S}^{2}$ such that $x \mapsto d(x, C)$ is not convex on any neighborhood of $C$. Practical interest for quadratic "morphological lipschitzation" of surfaces or images valued on surfaces should be explored in ongoing work.

\section{Generalized Riemannian morphological operators}

We have discussed the canonic case on Riemannian mathematical morphology associated to the structuring function $\mathfrak{q}_{\lambda}(x, y)$. Let consider now the most general family of Riemannian operators. We start by introducing the minimal properties that a Riemannian structuring function should verify.

Definition 10. Let $\mathcal{M}$ be a Riemannian manifold. A mapping $\mathfrak{b}: \mathcal{M} \times \mathcal{M} \rightarrow \mathbb{R}$ defined for any pair of points in $\mathcal{M}$ is said an admissible Riemannian structuring function in $\mathcal{M}$ if and only if

1. $\mathfrak{b}(x, y) \leq 0, \forall x, y \in \mathcal{M}$ (non-positivity); 
2. $\mathfrak{b}(x, x)=0, \forall x \in \mathcal{M}$ (maximality at the diagonal).

Now, we can introduce the pair of dilation and erosion for any image $f$ according to $\mathfrak{b}$.

Definition 11. Given an admissible Riemannian structuring function $\mathfrak{b}$ in a Riemannian manifold $\mathcal{M}$, the Riemannian dilation and Riemannian erosion of an image $f \in \mathcal{F}(\mathcal{M}, \overline{\mathbb{R}})$ by $\mathfrak{b}$ are given respectively by

$$
\begin{aligned}
& \delta_{\mathfrak{b}}(f)(x)=\sup _{y \in \mathcal{M}}\{f(y)+\mathfrak{b}(x, y)\}, \\
& \varepsilon_{\mathfrak{b}}(f)(x)=\inf _{y \in \mathcal{M}}\{f(y)-\mathfrak{b}(y, x)\} .
\end{aligned}
$$

Note that this formulation has been considered recently in the framework of adaptive morphology (Ćurić and Luengo-Hendriks, 2013). Both are increasing operators which, by the maximality at the diagonal, preserves the extrema. By the non-positivity, Riemannian dilation is extensive and erosion is anti-extensive. In addition, we can easily check that the pair $\left(\varepsilon_{\mathfrak{b}}, \delta_{\mathfrak{b}}\right)$ forms an adjunction as in Proposition 3. Consequently, their composition leads to the Riemannian opening and closing according to the admissible Riemannian structuring function $\mathfrak{b}$ given respectively by:

$$
\begin{aligned}
\gamma_{\mathfrak{b}}(f)(x) & =\sup _{z \in \mathcal{M}} \inf _{y \in \mathcal{M}}\{f(y)-\mathfrak{b}(y, z)+\mathfrak{b}(z, x)\}, \\
\varphi_{\mathfrak{b}}(f)(x) & =\inf _{z \in \mathcal{M}} \sup _{y \in \mathcal{M}}\{f(y)+\mathfrak{b}(z, y)-\mathfrak{b}(x, z)\} .
\end{aligned}
$$

Remarkably, the symmetry of $\mathfrak{b}$ is not a necessary condition for the adjunction. Examples of such asymmetric structuring functions have recently appeared in the context of stochastic morphology (Angulo and Velasco-Forero, 2013), non-local morphology (Velasco-Forero and Angulo, 2013) and saliency-based adaptive morphology (Ćurić and Luengo-Hendriks, 2013).

In our framework, we propose a general form of any admissible Riemannian structuring function $\mathfrak{b}(x, y), \forall x, y \in \mathcal{M}$, which should be decomposable into the sum of two terms:

$$
\mathfrak{b}(x, y)=\alpha \mathfrak{b}^{\text {sym }}(x, y)+\beta \mathfrak{b}^{\text {asym }}(x, y), \quad \alpha, \beta \geq 0 .
$$

Symmetric structuring function. The symmetric term of the structuring function will be a scaled p-norm shaped function depending exclusively on the Riemannian distance, i.e., $\mathfrak{b}^{\text {sym }}(x, y)=\mathfrak{b}^{\text {sym }}(y, x)=k_{\lambda, p}\left(d_{\mathcal{M}}(x, y)\right)$ such that

$$
k_{\lambda, p}(\eta)=-C_{p} \frac{\eta^{\frac{p}{p-1}}}{\lambda^{\frac{1}{p-1}}} ; \lambda>0, \quad p>1,
$$

where the normalization factor is given by $C_{p}=(p-1) p^{-\frac{p}{p-1}}$. We note that with the shape parameter $p=2$ we recover the canonic quadratic structuring function. In fact, this generalization of the quadratic structuring is inspired from the solution of a generalized morphological PDE (Lions et al., 1987): $u_{t}(t, x)+\left\|u_{x}(t, x)\right\|^{p}=0,(t, x) \in(0,+\infty) \times E ; u(0, x)=f(x)$, $x \in E$, since the quadratic one is the solution of the classical (Hamilton-Jacobi) morphological PDE (Bardi et al., 1984; Crandall et al., 1992): $u_{t}(t, x)+\left\|u_{x}(t, x)\right\|^{2}=0$. Asymptotically, one is dealing with almost flat shapes over $\mathcal{M}$ as $p \rightarrow 1$; as $p>2$ increases and $p \rightarrow \infty$ the shape of $k_{\lambda, p}(\eta)$ evolves from a parabolic shape $p=2$, i.e., term on $d_{\mathcal{M}}(x, y)^{2}$, to the limit case, which is a conic shape, i.e., term on $d_{\mathcal{M}}(x, y)$.

We note that if $\mathcal{M}$ is a Cartan-Hadamard manifold, the symmetric part $\mathfrak{b}^{s y m}(x, y)$ is a concave function for any $\lambda>0$ and any $p>1$.

Asymmetric structuring function. Relevant forms of the asymmetric term is an open issue on Riemannian morphology, which will probably allows to introduce more advanced morphological operators. For instance, we can fix a reference point $o \in \mathcal{M}$ and define, for $x, y \in \mathcal{M}, y \neq o$, the function

$$
\mathfrak{b}_{\lambda, o}^{a s y m}(x, y)=-\frac{d_{\mathcal{M}}(x, y)}{\lambda d_{\mathcal{M}}(y, o)}
$$

The assignment $x \mapsto \mathfrak{b}_{\lambda, o}^{a s y m}(x, y)$ involves a shape strongly deformed near the reference point. One can also replace the reference point by a set $O \subset \mathcal{M}$, hence changing $d_{\mathcal{M}}(y, o)$ by the distance function $d_{\mathcal{M}}(y, O)$.

An alternative asymmetric function could be based on the notion of Busemann function (Ballmann et al., 1985). Given a point $x \in \mathcal{M}$ and a ray $\gamma$ starting at $x$ in the direction of the tangent vector $\mathbf{v}$, i.e., a unit-speed geodesic line $\gamma:[0, \infty)$ $\rightarrow \mathcal{M}$ such that $d_{\mathcal{M}}(\gamma(0), \gamma(t))=t$ for all $t \geq 0$, one defines its Busemann function $b_{\gamma_{x, v}}$ by the formula

$$
\begin{aligned}
b_{\gamma_{x, \mathbf{v}}}(y) & =\lim _{t \rightarrow \infty}\left[d_{\mathcal{M}}\left(x, \gamma_{x, \mathbf{v}}(0)\right)-d_{\mathcal{M}}\left(y, \gamma_{x, \mathbf{v}}(t)\right)\right] \\
& =\lim _{t \rightarrow \infty}\left[t-d_{\mathcal{M}}\left(y, \gamma_{x, \mathbf{v}}(t)\right)\right]
\end{aligned}
$$

Since $t-d_{\mathcal{M}}\left(y, \gamma_{x, \mathbf{v}}(t)\right)$ is bounded above by $d_{\mathcal{M}}\left(x, \gamma_{x, \mathbf{v}}(0)\right)$ and is monotone non-decreasing in $t$, the limit always exists. It follows that $\left|b_{\gamma_{x, v}}(y)-b_{\gamma_{x, v}}(z)\right| \leq d_{\mathcal{M}}(y, z)$, i.e., Busemann function is Lipschitz with constant 1 . If $\mathcal{M}$ has non-negative sectional curvature $b_{\gamma_{x, v}}(y)$ is convex. If $\mathcal{M}$ is Cartan-Hadamard manifold, it is concave. Consequently, we can define our asymmetric structuring function as

$$
\mathfrak{b}_{\lambda, \mathbf{v}}^{a s y m}(x, y)= \begin{cases}-\lambda^{-1} b_{\gamma_{x, \mathbf{v}}}(y) & \text { if sect. curvature of } \mathcal{M} \geq 0 \\ \lambda^{-1} b_{\gamma_{x, \mathbf{v}}}(y) & \text { if sect. curvature of } \mathcal{M}<0\end{cases}
$$

From a practical viewpoint, asymmetric structuring functions obtained by Busemann function allow to introduce a shape which depends on the distance between the point $x$ and a kind of orthogonal projection of point $y$ on the geodesic along the direction $\mathbf{v}$. Hence, it could be a way to introduce directional Riemannian operators.

\section{Parallel transport of a fixed external structuring func- tion}

Previous Riemannian morphological operators are based on geodesic structuring functions $\mathfrak{b}(x ; y)$ which are defined by the geodesic distance function on $\mathcal{M}$. Let us consider now the case where a prior (semi-continuous) structuring function $b$ external to $\mathcal{M}$ is given and it should be adapted to each point $x \in \mathcal{M}$. Our approach is inspired from Roerdink (1994) formulation of dilation/erosion for binary images on smooth surfaces. 


\subsection{Manifold morphology}

The idea behind the binary Riemannian morphology on smooth surfaces introduced in (Roerdink, 1994) is to replace the translation invariance by the parallel transport (the transformations are referred to as "covariant" operations). Let $\mathcal{M}$ be a (geodesically complete) Riemannian manifold and $\mathcal{P}(\mathcal{M})$ denotes the set of all subsets of $M$. A binary image $X$ on the manifold is just $X \in \mathcal{P}(\mathcal{M})$. Let $A \subset \mathcal{M}$ be the basic structuring, a subset which is defined on the tangent space at a given point $\omega$ of $\mathcal{M}$ by $\tilde{A}=\log _{\omega}(A) \subset T_{\omega} \mathcal{M}$. Let $\gamma=\gamma_{[p, q]}$ be a path from $p$ to $q$, then the operator

$$
\tau_{\gamma}(A)=\operatorname{Exp}_{\mathrm{q}} P_{\gamma} \log _{\mathrm{p}}(A)=B,
$$

transports the subset $A$ of $p$ to the set $B$ of $q$. As the image of the set $X$ under parallel translation from $p$ to $q$ will depend in general on which path is taken; the solution proposed in (Roerdink, 1994), denoted by $\delta_{A}^{\text {Roerdink }}$, is to consider all possible paths from $p$ to $q$. The mapping $\delta_{A}^{\text {Roerdink }}: \mathcal{P}(\mathcal{M}) \rightarrow \mathcal{P}(\mathcal{M})$ given by

$$
\delta_{A}^{\text {Roerdink }}(X)=\bigcup_{x \in \mathcal{M}} \bigcup_{\gamma} \tau_{\gamma}(A)=\bigcup_{x \in \mathcal{M}} \bigcup_{\gamma} \operatorname{Exp}_{\mathrm{x}} P_{\gamma_{[\omega, x]}} \log _{\omega}(A),
$$

is a dilation of image $X$ according to the structuring element $A$. Using the symmetry group morphology (Roerdink, 2000), this operator can be rewritten as

$$
\delta_{A}^{\text {Roerdink }}(X)=\bigcup_{x \in \mathcal{M}} \operatorname{Exp}_{x} P_{\gamma_{[\omega, x]}} \log (\bar{A}),
$$

where $\bar{A}=\bigcup_{s \in \Sigma} s A$, with $\Sigma$ being the holonomy group around the normal at $\omega$. For instance, if $\tilde{A}=\log _{\omega}(A)$ is a line segment of length $r$ starting at $\omega$ then $\bar{A}$ is a disk of radius $r$ centered at $\omega$.

\section{2. $b_{\omega}$-transported Riemannian dilation and erosion}

Coming back to our framework of real-valued images on a geodesically complete Riemannian manifold $\mathcal{M}$. From our viewpoint, it seems more appropriate to fix the reference structuring element as a Boolean set $S$ on the tangent space at the reference point $\omega \in \mathcal{M}$, i.e., $S_{\omega} \subset T_{\omega} \mathcal{M}$. More precisely, let $S_{\omega}$ be a compact set which contains the origin of $T_{\omega} \mathcal{M}$. We can now formulate the $S_{\omega}$-transported flat Riemannian dilation and erosion as

$$
\begin{aligned}
& \breve{\delta}_{S_{\omega}}(f)(x)=\sup \left\{f(y): y \in \operatorname{Exp}_{\mathrm{x}} P_{\gamma_{[\omega, x]}^{\text {geo }}} \check{S}_{\omega}\right\}, \\
& \breve{\varepsilon}_{S_{\omega}}(f)(x)=\inf \left\{f(y): y \in \operatorname{Exp}_{\mathrm{x}} P_{\gamma_{[\omega, x]}^{\text {geo }}} S_{\omega}\right\} .
\end{aligned}
$$

Thus, in comparison to dilation (20), we prefer to consider in our case that the parallel transport from $\omega$ to $x$ is done exclusively along the geodesic path $\gamma_{[\omega, x]}^{g e o}$ between $\omega$ and $x$, i.e., if $S_{\omega}$ is a line in $\omega$ then it will be also at $x$ a line, but rotated.

This idea leads to a natural extension to the case where the fixed datum is an upper-semicontinuous structuring function $b_{\omega}(\mathbf{v})$, defined in the Euclidean tangent space at $\omega$, i.e., $b_{\omega}: T_{\omega} \mathcal{M} \rightarrow[-\infty, 0]$. Let consider now the upper level sets (or cross-section) of $b_{\omega}$ obtained by thresholding at a value $l$ :

$$
X_{l}\left(b_{\omega}\right)=\left\{\mathbf{v} \in T_{\omega} \mathcal{M}: b_{\omega}(\mathbf{v}) \geq l\right\}, \quad \forall l \in[-\infty, 0] .
$$

The set of upper level sets constitutes a family of decreasing closed sets: $l \geq m \Rightarrow X_{l} \subseteq X_{m}$ and $X_{l}=\cap\left\{X_{m}, m<l\right\}$. Any function $b_{\omega}(\mathbf{v})$ can be now viewed as an unique stack of its crosssections, which leads to the following reconstruction property:

$$
b_{\omega}(\mathbf{v})=\sup \left\{l \in[-\infty, 0]: \mathbf{v} \in X_{l}\left(b_{\omega}\right)\right\}, \quad \forall \mathbf{v} \in \mathcal{M} .
$$

Using this representation, the corresponding Riemannian structuring function at $\omega$ is given by $\mathfrak{b}_{\omega}(\omega, y)=\sup \{l \in[-\infty, 0]: z \in$ $\left.\operatorname{Exp}_{\omega} X_{l}\left(b_{\omega}\right)\right\}$. In the case of a different point $x \in \mathcal{M}$, the crosssection should be transported to the tangent space of $x$ before mapping back to $\mathcal{M}$, i.e.,

$$
\mathfrak{b}_{\omega}(x, y)=\sup \left\{l \in[-\infty, 0]: z \in \operatorname{Exp}_{\omega} P_{\gamma_{[\omega, x]}^{\text {geo }}} X_{l}\left(b_{\omega}\right)\right\} .
$$

Finally, the $b_{\omega}$-transported Riemannian dilation and erosion of image $f$ are given respectively by

$$
\begin{aligned}
& \breve{\delta}_{b_{\omega}}(f)(x)=\sup _{y \in \mathcal{M}}\left\{f(y)+\mathfrak{b}_{\omega}(x, y)\right\}, \\
& \breve{\varepsilon}_{b_{\omega}}(f)(x)=\inf _{y \in \mathcal{M}}\left\{f(y)-\mathfrak{b}_{\omega}(y, x)\right\} .
\end{aligned}
$$

Obviously, the case of a concave structuring function $b_{\omega}$ is particularly well defined since in such a case, its cross-sections are convex sets. In addition, if $\mathcal{M}$ is a Cartan-Hadamard manifold, the corresponding Riemannian structuring function $\mathfrak{b}_{\omega}(x, y)$ is also a concave function.

A typical useful case consists in taking at reference $\omega$ the structuring function:

$$
b_{\omega}(\mathbf{v})=-\frac{\mathbf{v}^{T} Q \mathbf{v}}{2}
$$

where $Q$ is a $d \times d$ symmetric positive definite matrix, $d$ being the dimension of manifold $\mathcal{M}$. It corresponds just to a generalized quadratic function such that the eigenvectors of $Q$ define the principal directions of the concentric ellipsoids and the eigenvalues their eccentricity. Therefore, we can introduce by means of $Q$ an anisotropic/directional shape on $\mathfrak{b}_{\omega}(x, y)$. We can easily check that $Q=\frac{1}{\lambda} I, I$ being the identity matrix of dimension $d$, corresponds just to the canonic Riemannian dilation and erosion (1) and (2).

Without an explicit expression of the exponential map, we cannot compute straightforward the $b_{\omega}$-transported Riemannian dilation and erosion on a Riemannian manifold $\mathcal{M}$. This is for instance the situation when is $f$ is an image on a 3D smooth surface. Hence, in the case of applications to valued surfaces, manifold learning techniques as LOGMAP (Brun et al., 2005) can be used to numerically obtain the transported cross-sections on $\mathcal{M}$.

\section{Connections with classical Euclidean morphology}

Riemannian dilation and erosion generalize previous operators in Euclidean morphology for both, translation invariant and spatially-variant morphology. 


\subsection{Spatially-invariant operators}

First of all, it is obvious that the Riemannian dilation/erosion naturally extends the quadratic Euclidean dilation/erosion for images $\mathcal{F}\left(\mathbb{R}^{d}, \overline{\mathbb{R}}\right)$ by considering that the intrinsic distance is the Euclidean one (or the discrete one for $\left.\mathbb{Z}^{d}\right)$, i.e., $d_{\mathcal{M}}(x, y)=$ $\|x-y\|=d_{\text {space }}(x, y)$.

By the way, we note also that definition of the Riemannian flat dilation and erosion of size $r$ given in (8) and (9) are compatible with the formulation of the classical geodesic dilation and erosion (Lantuejoul and Beucher, 1981) of size $r$ of image $f$ (marker) constrained by the image $g$ (reference or mask), $\delta_{g, \lambda}(f)$ and $\varepsilon_{g, \lambda}(f)$, which underly the operators by reconstruction (Soille, 1999), where the upper-level sets of the reference image $g$ are considered as the manifold $\mathcal{M}$ where the geodesic distance is defined.

\subsection{Adaptive (spatially-variant) operators}

From (Kimmel et al., 1997), the idea of embedding a 2D grey-level image $f \in \mathcal{F}\left(\mathbb{R}^{2}, \overline{\mathbb{R}}\right), x=\left(x_{1}, x_{2}\right)$, into a surface embedded in $\mathbb{R}^{3}$, i.e.,

$$
f(x) \mapsto \xi_{x}=\left(x_{1}, x_{2}, \alpha f\left(x_{1}, x_{2}\right)\right), \alpha>0,
$$

where $\alpha$ is a scaling parameter useful for controlling intensity distances, has become popular in differential geometry inspired image processing. This embedded Riemannian manifold $\mathcal{M}=$ $\mathbb{R}^{2} \times \overline{\mathbb{R}}$ has a product metric of type $d s_{\mathcal{M}}^{2}=d s_{\text {space }}^{2}+\alpha d s_{f}^{2}$, where $d s_{\text {space }}^{2}=d x_{1}^{2}+d x_{2}^{2}$ and $d s_{f}^{2}=d f^{2}$. The geodesic distance between two points $\xi_{x}, \xi_{y} \in \mathcal{M}$ is the length of the shortest path between the points, i.e., $d_{\mathcal{M}}\left(\xi_{x}, \xi_{y}\right)=\min _{\gamma=\gamma_{\left[\xi_{x}, \xi_{y}\right]}} \int_{\gamma} d s_{\mathcal{M}}$.

As shown in (Welk et al., 2011), this is essentially the framework behind the morphological amoebas (Lerallut et al., 2007), which are flat spatially adaptive structuring functions centered in a point $x, A_{\lambda}(x)$, computed by thresholding the geodesic distance at radius $\lambda>0$, i.e., $A_{\lambda}(x)=\left\{y \in E: d_{\mathcal{M}}\left(\xi_{x}, \xi_{y}\right)<\lambda\right\}$. In the discrete setting, the geodesic distance is given by

$$
\begin{aligned}
d_{\mathcal{M}}\left(\xi_{x}, \xi_{y}\right)= & \min _{\left\{\xi^{1}=\xi_{x}, \xi^{2}, \cdots, \xi^{N}=\xi_{y}\right\}} \sum_{i=1}^{N} \alpha\left|f\left(x^{i}\right)-f\left(x^{i+1}\right)\right| \\
& +\sqrt{\left(x_{1}^{i}-x_{1}^{i+1}\right)^{2}+\left(x_{2}^{i}-x_{2}^{i+1}\right)^{2}} .
\end{aligned}
$$

We should remark that for $x \rightarrow y$ and assuming a smooth manifold, the geodesic distance is asymptotically equivalent to the corresponding distance in the Euclidean product space, i.e.,

$$
d_{\mathcal{M}}\left(\xi_{x}, \xi_{y}\right)^{2} \approx d_{\text {space }}(x, y)^{2}+\alpha^{2}|f(x)-f(y)|^{2},
$$

which is the distance appearing in the bilateral structuring functions (Angulo, 2013). We can also see that the salience maps behind the salience adaptive structuring elements (Ćurić et al., 2012) can be approached in a Riemannian formulation by choosing the appropriate metric.

\section{Various useful case studies}

We state in this Section several interesting cases of image manifolds (and their corresponding Riemannian distances) which can be processed using Riemannian morphological operators.

\subsection{Hyperbolic embedding of an Euclidean positive image into Poincaré half-space $\mathcal{H}^{3}$}

Shortest path distance (27) is not invariant to scaling of image intensity, i.e., $f \mapsto f^{\prime}=\beta f, \beta>0$ involves that $\mid f^{\prime}\left(x^{i}\right)-$ $f^{\prime}\left(x^{i+1}\right)|=\beta| f\left(x^{i}\right)-f\left(x^{i+1}\right) \mid$ and hence the shape of the corresponding Riemannian structuring function for $f$ and $f^{\prime}$ will be different. This lack of contrast invariance can be easily solved by using a logarithmic metric in the intensities. Hence, if we assume positive intensities, $f(x)>0$, for all $x \in \mathcal{M}$, we can consider the distance $d_{\mathcal{M}}\left(\xi_{x}, \xi_{y}\right)=\min _{\gamma_{\xi x}, \xi_{y}} \sum_{i=1}^{N} d_{\text {space }}\left(x^{i}, x^{i+1}\right)+$ $\alpha\left|\log f\left(x^{i}\right)-\log f\left(x^{i+1}\right)\right|$. This metric can be connected to the logarithmic image processing (LIP) model (Jourlin and Pinoli, 1988). This geometry can be also justified from a human perception viewpoint. The classical Weber-Fechner law states that human sensation is proportional to the logarithm of the stimulus intensity. In the case of vision, the eye senses brightness approximately according to the Weber-Fechner law over a moderate range.

Following the same assumption of positive intensities, we can also consider that a 2D image can be embedded into the hyperbolic space $\mathcal{H}^{3}$ (Cannon et al., 1997). More particularly the (Poincaré) upper half-space model is the domain $\mathcal{H}^{3}=$ $\left\{\left(x_{1}, x_{2}, x_{3}\right) \in \mathbb{R}^{3} \mid x_{3}>0\right\}$ with the Riemannian metric $d s_{\mathcal{H}^{3}}^{2}=$ $\frac{d x_{1}^{2}+d x_{2}^{2}+d x_{3}^{2}}{x_{3}^{2}}$. This space has constant negative sectional curvature. If we consider the image embedding $f(x) \mapsto \xi_{x}=\left(x_{1}, x_{2}, f\left(x_{1}, x_{2}\right)\right)$ $\mathcal{H}^{3}$, the Riemannian distance needed for morphological operators will be given by

$$
d_{\mathcal{M}}\left(\xi_{x}, \xi_{y}\right)=\min _{\gamma_{\xi_{x}, \xi_{y}}} \sum_{i=1}^{N} \cosh ^{-1}\left(1+\frac{\left(x_{1}^{i}-x_{1}^{i+1}\right)^{2}+\left(x_{2}^{i}-x_{2}^{i+1}\right)^{2}+\left(f\left(x^{i}\right)-f\left(x^{i+1}\right)^{2}\right.}{2 f\left(x^{i}\right) f\left(x^{i+1}\right)}\right) .
$$

The geometry of this space is extremely rich in particular concerning the invariance and isometric symmetry. Hence, distance (29) is for instance invariant to translations $\xi=\left(x_{1}, x_{2}, x 3\right)$ $\mapsto \xi+\alpha, \alpha \in \mathbb{R}$, scaling $\xi \mapsto \beta \xi, \beta>0$. A specific theory on granulometric scale-space properties in this embedding can be intended.

\subsection{Embedding an Euclidean image into the structure tensor manifold}

Besides the space $\times$ intensity embeddings discussed just above, we can consider other more alternative non-Euclidean geometric embedding of scalar images, using for instance the local structure.

More precisely, given a 2D Euclidean image $f(x)=f\left(x_{1}, x_{2}\right)$ $\in \mathcal{F}\left(\mathbb{R}^{2}, \overline{\mathbb{R}}\right)$, the structure tensor representing the local orientation and edge information (Förstner and Gülch, 1987) is obtained by Gaussian smoothing of the dyadic product $\nabla f \nabla f^{T}$ :

$S(f)(x)=G_{\sigma} *\left(\nabla f\left(x_{1}, x_{2}\right) \nabla f\left(x_{1}, x_{2}\right)^{T}\right)=\left(\begin{array}{cc}s_{x_{1} x_{1}}\left(x_{1}, x_{2}\right) & s_{x_{1}, x_{2}}\left(x_{1}, x_{2}\right) \\ s_{x_{1} x_{2}}\left(x_{1}, x_{2}\right) & s_{x_{2} x_{2}}\left(x_{1}, x_{2}\right)\end{array}\right)$

where $\nabla f\left(x_{1}, x_{2}\right)=\left(\frac{\partial f\left(x_{1}, x_{2}\right)}{\partial x_{1}}, \frac{\partial f\left(x_{1}, x_{2}\right)}{\partial x_{2}}\right)^{T}$ is the 2D spatial intensity gradient and $G_{\sigma}$ stands for a Gaussian smoothing with a standard deviation of $\sigma$. From a mathematical viewpoint, $S(f)(x)$ : $E \rightarrow \operatorname{SPD}(2)$ is an image where at each pixel we have a symmetric positive definite matrix $2 \times 2$. The differential geometry in the manifold $\operatorname{SPD}(n)$ is very well-known (Bhatia, 2007). 
(b)

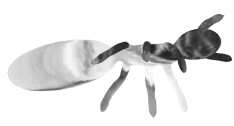

(a)

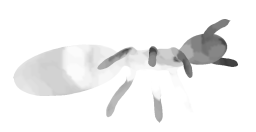

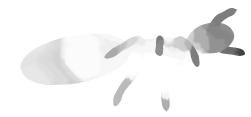

(c)

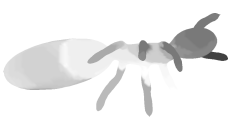

(e)

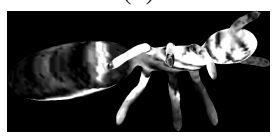

(g) (f)

(d)

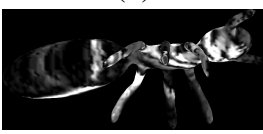

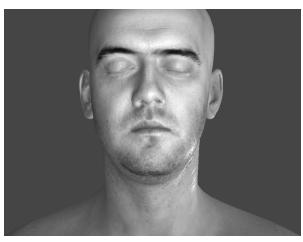

(a)

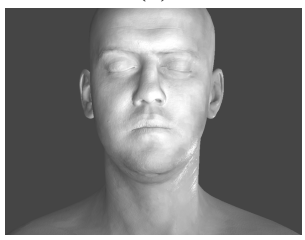

(c)

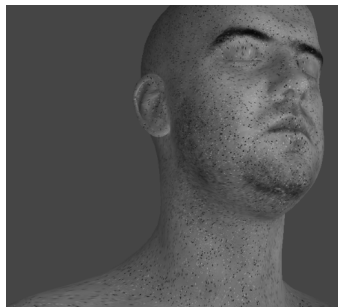

(e)

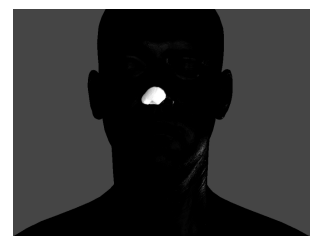

(b)

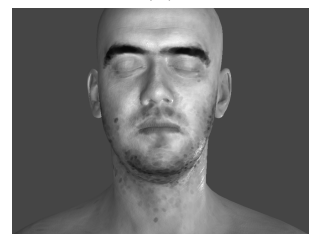

(d)

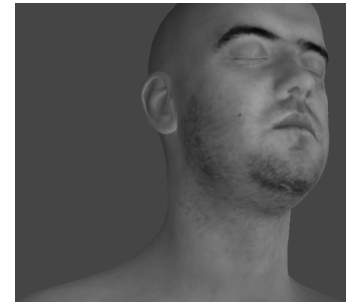

(f) and the Riemannian distance is defined as $d_{S P D(n)}\left(M_{1}, M_{2}\right)=$ $\left\|\log \left(M_{1}^{-1 / 2} M_{2} M_{1}^{-1 / 2}\right)\right\|_{F}, \forall M_{1}, M_{2} \in \operatorname{SPD}(n)$. Let consider now the embedding $f(x) \mapsto \xi_{x}=\left(x_{1}, x_{2}, \alpha S(f)\left(x_{1}, x_{2}\right)\right), \alpha>0$, in the product manifold $\mathcal{M}=\mathbb{R}^{2} \times \operatorname{SPD}(2)$, which has the product metric $d s_{\mathcal{M}}^{2}=d s_{\text {space }}^{2}+\alpha d s_{S P D(2)}^{2}$. It is a (complete, not compact, negative sectional curved) Riemannian manifold of geodesic distance given by $d_{\mathcal{M}}\left(\xi_{x}, \xi_{y}\right)=\min _{\gamma_{\xi_{x}, \xi_{y}}} \sum_{i=1}^{N} d_{\text {space }}\left(x^{i}, x^{i+1}\right)^{\text {ers. }}$

$+\alpha d_{S P D(n)}\left(S(f)\left(x^{i}\right), S(f)\left(x^{i+1}\right)\right)$, which is asymptotically equal to $d_{\mathcal{M}}\left(\xi_{x}, \xi_{y}\right)^{2} \approx d_{\text {space }}(x, y)^{2}+\alpha d_{S P D(2)}(S(f)(x), S(f)(y))^{2}$.

By means of this embedding, we can compute anisotropic morphological operators following the flow coherence of image structures. This embedding is connection to previous adaptive approaches such as (Verdú et al., 2011) and (Landström and Thurley, 2013).

\subsection{Embedding star-like $3 D$ surfaces into the sphere $\mathbb{S}^{2}$}

Let consider a smooth surface $\mathcal{S} \subset \mathbb{R}^{3}$ under the assumption that it corresponds to a star-shaped 3D objet: that means that there exists a point $z_{0} \in \mathbb{R}^{3}$ within the object, such that each ray originating from this point intersects the object's surface exactly one; this point is denoted $\pi_{z_{0} \rightarrow \mathcal{S}}$. We can assign to each ray, representing a 3D orientation in space and therefore an element of the sphere $\mathbb{S}^{2}$, the distance of the ray-surfaceintersection to the origin of the ray. Hence, we have a function $f: \mathbb{S}^{2} \rightarrow \mathbb{R}_{+}$, where $\left(\mathcal{S}, z_{0}\right) \mapsto f(\theta, \phi)=\left\|z_{0}-\pi_{z_{0} \rightarrow \mathcal{S}}\right\|,(\theta, \phi)$ are the standard spherical coordinates. The Riemannian geodesic distance in $\mathbb{S}^{2}$ (or Great circle distance) is simple the angle between the two points; i.e., $d_{\mathbb{S}^{2}}\left(\xi_{1}, \xi_{2}\right)=\arccos \left(\xi_{1} \cdot \xi_{2}\right)$, where $\xi_{i}=\left(\sin \theta_{i} \cos \varphi_{i}, \sin \theta_{i} \sin \varphi_{i}, \cos \theta_{i}\right)$. The image $f$ can be processed using the Riemannian morphological operators, obtaining the image $f^{\prime}$ and the corresponding processed surface $\mathcal{S}^{\prime}$ is simply obtained by inversion of projective mapping $\pi_{z_{0} \rightarrow \mathcal{S}^{\prime}}$.
Figure 3: Morphological processing of real valued 3D surface of a face: (a) original image on a surface $\mathcal{S} \subset \mathbb{R}^{3}, f(x) \in \mathcal{F}\left(\mathcal{S}, \mathbb{R}_{+}\right)$; (b) example of geodesic ball $B_{r}(x)$ at a given point $x \in \mathcal{S}$; (d) and (e) Riemannian dilation $\delta_{\lambda}(f)(x)$ and Riemannian erosion $\varepsilon_{\lambda}(f)(x)$ with $\lambda=0.5$; (e) nonsmooth version of surface (added impulse noise); (f) filtered surface obtained by Lasry-Lions regulariz-

\subsection{Morphological processing of real valued $3 D$ surfaces}

In Fig. 2(a) is given an example of real-valued 3D surface, i.e., the image to be processed is $f: \mathcal{S} \subset \mathbb{R}^{3} \rightarrow \overline{\mathbb{R}}$. In practice, the $3 \mathrm{D}$ surface is represented by a mesh (i.e., triangulated manifold with a discrete structure composed of vertices, edges and faces). In our example, the grey-level intensities are supported on the vertices. In the case of a discrete approximation of a manifold based on mesh representation, the geodesic distance $d_{\mathcal{S}}(x, y)$ can be calculated by the Floyd-Warshall algorithm for finding shortest path in the weighted graph of vertices of the mesh. Efficient algorithms are based on Fast Marching generalized to arbitrary triangulations Kimmel and Sethian (1998). Fig. 2 depicts examples of Riemannian dilation $\delta_{\lambda}(f)$ and Riemannian closing $\varphi_{\lambda}(f)$, for two different scales $(\lambda=4$ and $\lambda=8$ ) and the corresponding dual top-hats.

Another example of real valued surface is given in Fig. 3. It corresponds to the 3D acquisition of a face. We observe how the canonic Riemannian dilation and erosion are able to locally process the face details taking into the geometry of the surface. In Fig. 3 is also given an example of image filtering using the composition our Lasry-Lions regularizers (15) (with $\lambda=4$ and $\mu=2$ ), where the original surface is a nonsmooth version obtained by adding impulse noise. 


\section{Conclusions}

We have introduced in this paper a general theory for the formulation of mathematical morphology operators for images valued on Riemannian manifolds. We have defined the main operators and studied their fundamental properties.

We have considered two main families of operators. On the one hand, morphological operators based on an admissible Riemannian structuring function which is adaptively obtained for each point $x$ according to the geometry of the manifold. On the other hand, morphological operators founded on an external Euclidean structuring function which is parallel transported to the tangent space at each point $x$ and then mapped to the manifold.

We have also discussed some original Riemannian embedding of Euclidean images onto Cartan-Hadamard manifolds. This is the case of the Poincaré half-space $\mathcal{H}^{3}$ as well as the structure tensor manifold. Riemannian structuring functions defined on Cartan-Hadamard manifolds are particular rich in terms of scale-space properties as well as in Lipschitz regularization.

\section{References}

J. Angulo. Morphological Bilateral Filtering. SIAM Journal on Imaging Sciences, To appear, 2013.

J. Angulo and S. Velasco-Forero. Mathematical morphology for real-valued images on Riemannian manifolds. In Proc. of ISMM'13 (11th International Symposium on Mathematical Morphology), Springer LNCS 7883, p. 279291, 2013.

J. Angulo and S. Velasco-Forero. Stochastic Morphological Filtering and Bellman-Maslov Chains. In Proc. of ISMM'13 (11th International Symposium on Mathematical Morphology), Springer LNCS 7883, p. 171-182, 2013.

D. Attouch, D. Aze. Approximation and regularization of arbitray functions in Hilbert spaces by the Lasry-Lions method. Annales de l'I.H.P., section C, 10(3): 289-312, 1993.

D. Azagra, J. Ferrera. Inf-Convolution and Regularization of Convex Functions on Riemannian Manifols of Nonpositive Curvature. Rev. Mat. Complut. 19(2): 323-345, 2006.

W. Ballmann, M. Gromov, V. Schroeder. Manifolds of nonpositive curvature. Progr. Math., 61, Birkhäuser, 1985.

M. Bardi, L.C. Evans. On Hopf's formulas for solutions of Hamilton- Jacobi equations. Nonlinear Analysis, Theory, Methods and Applications, 8(11):1373-1381, 1984.

R. Bhatia. Positive Definite Matrices. Princeton University Press, 2007.

M. Berger, B. Gostiaux. Differential Geometry: Manifolds, Curves, and Surfaces. Springer, 1987.

R. van den Boomgaard, L. Dorst. The morphological equivalent of Gaussian scale-space. In Proc. of Gaussian Scale-Space Theory, 203-220, Kluwer, 1997.

A. Brun, C.-F. Westin, M. Herberthson, H. Knutsson. Fast Manifold Learning Based on Riemannian Normal Coordinates. In Proc. of 14th Scandinavian Conference (SCIA'05), Springer LNCS 3540, 920-929, 2005.

J.W. Cannon, W.J. Floyd, R. Kenyon, W.R. Parry. Hyperbolic Geometry. Flavors of Geometry, MSRI Publications, Vol. 31, 1997.

M.G. Crandall, H. Ishii, P.-L. Lions. User's guide to viscosity solutions of second order partial differential equations. Bulletin of the American Mathematical Society, 27(1):1-67, 1992.

V. Curić, C.L. Luengo Hendriks, G. Borgefors. Salience adaptive structuring elements. IEEE Journal of Selected Topics in Signal Processing, 6(7): 809$819,2012$.

V. Curić and C.L. Luengo-Hendriks. Salience-Based Parabolic Structuring Functions. In Proc. of ISMM'13 (11th International Symposium on Mathematical Morphology), Springer LNCS 7883, p. 183-194, 2013.
J. Debayle and J. C. Pinoli. Spatially Adaptive Morphological Image Filtering using Intrinsic Structuring Elements. Image Analysis and Stereology, 24(3):145-158, 2005.

W. Förstner, E. Gülch. A fast operator for detection and precise location of distinct points, corners and centres of circular features. In Proc. of ISPRS Intercommission Conference on Fast Processing of Photogrammetric Data, p. 281-304, 1987.

H.J.A.M. Heijmans. Morphological image operators. Academic Press, Boston, 1994.

P.T. Jackway, M. Deriche. Scale-Space Properties of the Multiscale Morphological Dilation-Erosion. IEEE Trans. Pattern Anal. Mach. Intell., 18(1): 38 $51,1996$.

M. Jourlin, J.C. Pinoli. A model for logarithmic image processing. Journal of Microscopy, 149(1):21-35, 1988.

R. Kimmel, N. Sochen, R. Malladi. Images as embedding maps and minimal surfaces: movies, color, and volumetric medical images. In Proc. of IEEE CVPR'97, pp. 350-355, 1997.

R. Kimmel, J.A. Sethian. Computing geodesic paths on manifolds. Proc. of National Academy of Sci., 95(15): 8431-8435, 1998.

C. Kiselman. Division of mappings between complete lattices. In Proc. of the 8th International Symposium on Mathematical Morphology (ISMM'07), Rio de Janeiro, Brazil, MCT/INPE, vol. 1, p. 27-38.

E.J. Kraus, H.J.A.M. Heijmans, E.R. Dougherty. Gray-scale granulometries compatible with spatial scalings. Signal Processing, 34(1): 1-17, 1993.

A. Landström, M.J. Thurley. Adaptive morphology using tensor-based elliptical structuring elements. Pattern Recognition Letters, 34(12): 1416-1422, 2013.

S. Lang. Fundamentals of differential geometry. Springer-Verlag, 1999.

C. Lantuejoul, S. Beucher. On the use of the geodesic metric in image analysis. Journal of Microscopy, 121(1): 39-49, 1981.

J.M. Lasry, P.-L. Lions. A remark on regularization in Hilbert spaces. Israel Journal of Mathematics, 55: 257-266, 1986

R. Lerallut, E. Decencière, F. Meyer. Image filtering using morphological amoebas. Image and Vision Computing, 25(4): 395-404, 2007.

P.-L. Lions, P.E. Souganidis, J.L. Vásquez. The Relation Between the Porous Medium and the Eikonal Equations in Several Space Dimensions. Revista Matemática Iberoamericana, 3 : 275-340, 1987.

P. Maragos. Slope Transforms: Theory and Application to Nonlinear Signal Processing. IEEE Trans. on Signal Processing, 43(4): 864-877, 1995.

G. Matheron. Random sets and integral geometry. John Wiley \& Sons, 1975.

J.J. Moreau. Fonctionnelles convexes. Lecture notes, Collège de France, Paris, 1967. Availible online in http://archive.numdam.org.

J.B.T.M. Roerdink. Manifold shape: from differential geometry to mathematical morphology. In Shape in Picture, NATO ASI F 126, pp. 209-223, Springer, 1994.

J.B.T.M. Roerdink. Group morphology. Pattern Recognition, 33: 877-895, 2000.

J.B.T.M. Roerdink. Adaptivity and group invariance in mathematical morphology. In Proc. of ICIP'09, 2009.

J. Serra. Image Analysis and Mathematical Morphology, Academic Press, London, 1988

J. Serra. Image Analysis and Mathematical Morphology. Vol II: Theoretical Advances, Academic Press, London, 1988.

P. Soille. Morphological Image Analysis, Springer-Verlag, Berlin, 1999.

S. Velasco-Forero and J. Angulo. On Nonlocal Mathematical Morphology. In Proc. of ISMM'13 (11th International Symposium on Mathematical Morphology), Springer LNCS 7883, p. 219-230, 2013.

R. Verdú, J. Angulo and J. Serra.Anisotropic morphological filters with spatially-variant structuring elements based on image-dependent gradient fields. IEEE Trans. on Image Processing, 20(1): 200-212, 2011.

M. Welk, M. Breuß, O. Vogel. Morphological amoebas are self-snakes. Journal of Mathematical Imaging and Vision, 39(2):87-99, 2011. 